\title{
Differentiation-inducing therapeutic effect of Notch inhibition in reversing malignant transformation of liver normal stem cells via MET
}

\author{
Hao Luo ${ }^{1,2, *}$, Wei-Hui Liu ${ }^{2, *}$, Hong-Yin Liang ${ }^{2, *}$, Hong-Tao Yan ${ }^{2, *}$, Ning Lin ${ }^{3}$, Dong-Yu \\ $\mathbf{L i}^{2}$, Tao Wang ${ }^{2, *}$ and Li-Jun Tang ${ }^{1,2}$ \\ ${ }^{1}$ Third Military Medical University, Chongqing 400038, China \\ ${ }^{2}$ General Surgery Center, Chengdu Military General Hospital, Chengdu 610083, China \\ ${ }^{3}$ Department of Clinical Nutrition, Chengdu Military General Hospital, Chengdu 610083, China \\ *These authors contributed equally to this work \\ Correspondence to: Li-Jun Tang, email: whitlj@163.com \\ Tao Wang, email: wangtao301020@sina.com \\ Keywords: liver cancer stem cells; liver normal stem cells; malignant transformation; Notch inhibition; differentiation inducing \\ therapy \\ Received: August 23, 2017 Accepted: January 01, 2018 Epub: February 05, 2018 Published: April 10, 2018 \\ Copyright: Luo et al. This is an open-access article distributed under the terms of the Creative Commons Attribution License 3.0 \\ (CC BY 3.0), which permits unrestricted use, distribution, and reproduction in any medium, provided the original author and source \\ are credited.
}

\section{ABSTRACT}

Background: Liver cancer stem cells (LCSCs) are the key factors for cancer metastasis, recurrent, and drug resistance. LCSCs are originated from either hepatocytes dedifferentiation or differentiation arresting of liver normal stem cells (LNSCs). Differentiation-inducing therapy is a novel strategy in solid tumors. Furthermore, Notch signaling pathway has been proved to play important role in the process of hepatocytes differentiation. In previous study, a malignant transformation cellular model of LNSCs has been built up, and in this study we are trying to illustrate whether inhibition of Notch can reverse this malignant tendency and drive these malignant cells back to differentiate into mature hepatocytes.

Results: Inhibition of Notch signaling pathway can down-regulate the stemnessrelated cancer markers, lower the proliferative status, alleviate the invasive characteristic, or attenuate the metastasis tendency. What is more, it can help the malignantly transformed cells to regain the mature hepatic function of glucagon synthesis, urea metabolism, albumin production, and indocyanine-green (ICG) clearance.

Materials and Methods: HOX transcript antisense RNA (HOTAIR) expression was enhanced in LNSCs via lentivirus transduction to set up the malignant transformation cellular model. Then, a Notch inhibitor was applied to induce malignantly transformed cells differentiate into mature hepatocytes, and malignant abilities of proliferation, invasiveness, tumorigenesis as well as mature hepatocyte function were observed and compared.

Conclusions: The data demonstrate that the anti-tumor effects of Notch inhibition may lie not only on killing the cancer cells or LCSCs directly, it can also induce the LCSCs differentiation into mature hepatocytes via mesenchymal-epithelial transition (MET) progress or downgrade the malignancy. 


\section{INTRODUCTION}

Hepatocellular carcinoma (HCC) is one of the most common malignant tumors, with high progressive, invasive, and recurrent rate, as well as drug resistance. The theory of liver cancer stem cells (LCSCs) gives a reasonable explanation of these phenomena. The origination of LCSCs is still debated. It is believed that LCSCs originate from dedifferentiation of mature hepatocytes or differentiation arrest of liver normal stem cells (LNSCs). No matter which theory is more coincident with what happened in vivo, differentiation-inducing therapy can be an adoptable option targeted both dedifferentiation of mature hepatocyte and differentiation arrested of LNSCs.

Differentiation-inducing therapeutic effect of alltrans retinoic acid serves as a treatment for leukemia at first. However, with the recognition of cancer stem cells increasing, differentiation-inducing therapy is widely used in solid tumor such as glioblastomas, skin cancer, and lung cancer [1-3]. Meanwhile, many regimens which are used in leukemia such as all-trans retinoic acid has presented a differentiation inducing the therapeutic effect in hepatocarcinoma, as well as other medicine [4-7]. What is more, using hepatic corresponding differentiationdetermining transcription factor such as hepatocyte nuclear factor (HNF) families, which are absent in HCC, can induce hepatoma cells differentiation into mature hepatocytes [8]. Differentiation inducing therapy can reverse the malignant phenotypes, prevent LCSCs from progressing and metastasis, and promote normal hepatic function recovery.

Notch signaling pathway has been proved to play a crucial role in both hepatocytes differentiation and anticarcinogenesis. Notch signaling pathway can control the differentiation balance between hepatocytes and cholangiocytes [9]. In the previous studies, we find that inhibition of Notch signaling pathway acts as a hepatic differential effect of LNSCs [10,11]. Meanwhile, Kang et al report that Notch activation drives adipocyte dedifferentiation and tumorigenic transformation in mice [12], while Notch inhibition presents multiple antitumor effect such as enhancing the sensitivity of chemo-therapy, suppressed cell growth, inducing apoptosis, and increasing cellular death [13-15]. To sum up, there are two possibly mehcanisms behind these antitumor effect. The first one is that inhibition of Notch is able to kill the HCCs directly. The other is that Notch inhibition plays the antitumor effect undirectly via inducing liver cancer cells differentiation into mature hepatocytes. However, whether Notch signaling pathway inhibition can demonstrate the differentiation inducing effect in malignantly transformed LNSCs is still unknown. Herein, we are trying to illustrate the differentiation-inducing therapeutic role of Notch signaling pathway inhibition in reversing the malignant transformation of LNSCs and regaining the lost hepatic function, and additionally to find out the potential mechanism.

\section{RESULTS}

\section{Notch inhibition could reverses the high proliferative status of malignantly transformed LNSCs}

To exclude the proliferative status of cells in each group, cell proliferation was assessed by MTT and cellular cycle protein was detected by western-blot. During the timeline of the cell growth assay, the numbers of cells in each group were similar in the first 3 day $(n=3, P>0.05)$. However, HOTAIR enhanced LNSCs were associated with a highest proliferative status after 3 days incubation $(n=3$, $P<0.05)$. Meanwhile, DAPT-induction could slow down accelerated proliferative speed of HOTAIR-enhanced cells $(n=3, P<0.05)$. What is more, both DAPT-induced and control cells presented a similar proliferative status, and the differences were not significant $(n=3, P>0.05)$ (Figure 1A).

WB results showed that the cellular cycle gene Cyclin D1 and proliferative gene c-Myc, also known as an oncogene, would be downregulated in DAPT-induction or under-expressed in normal cells spontaneously $(n=3$, $P<0.05$ ) (Figure $1 \mathrm{~B}$ and $1 \mathrm{C}$ ). It indicated that DAPT could lead these uncontrolled cells back to normal proliferative status.

\section{Notch inhibition neutralizes the high invasive status of malignantly transformed LNSCs}

To address the change of cellular invasion and migration, we performed transwell assays and scratch wound healing assays. Similar as the result of a previous study, we found it that HOTAIR-enhance could make the LNSCs gain a higher invasion and migration ability. Although more DAPT-induced cells trespass through the ECM than LNSCs, these trespassed cells were significantly less than that HOTAIR-enhanced group $(n=3, P<0.05)$ (Figure 2A). It is demonstrated DAPT-induction could partly neutralize the invasion ability of malignantly transformed cells. However, through the scratch wound healing assays, the gained migration ability of HOTAIRenhanced cells could be absolutely counteracted by DAPT inducing $(n=3, P<0.001)$ (Figure 2B).

\section{Notch inhibition drives malignantly transformed LNSCs into mature hepatocytes}

To address whether DAPT-induction promotes malignantly transformed LNSCs differentiation into mature hepatocytes, the excretory, synthetic, and metabolic function were evaluated. ICG was broadly used to evaluate the liver function. Herein, it was found that all three kinds 
of cells could intake ICG to the cytoplasm (Figure 3A). The difference was that HOTAIR-enhanced cells were able to absolutely eliminate the dye after about 1 hour. Quite differently, DAPT-induction could significantly shorten the elimination time to the level about 30 minutes, which was similar with normal LNSCs $(n=3, P<0.05)$ (Figure 3A).

The PAS staining was used to illustrate the change of synthetic function. the number of PAS-positive cells in HOTAIR group was 14/HPF, 29/HPF in DAPTinduction group $(n=10, P<0.05)$, and $16 / \mathrm{HPF}$ in LNSCs respectively (Figure 3B).

To demonstrate the difference of metabolic function of each group, the immature hepatic marker AFP, immature cholangiocytes marker CK7, and mature hepatic marker CYP as well as Alb were calculated using qRT-PCR. The Urea production, Alb production and Alt production were analyzed using an automatic biochemical analyzer. The lowest expression sample was set up as baseline. Immature hepatic marker AFP was 3.3-fold and 1.8-fold higher than that in DAPT-induction and LNSCs respectively $(n=3, P<0.05)$. Because LNSCs were bipotential cells which presents hepatic markers as well as cholangiocytic markers, CK-7 expression was highest expressed in LNSCs $(n=3, P<0.05)$. The patterns of mature hepatic marker, CYP and Alb expression were similar. DAPT-induced cells presents a highest expression of these mature hepatic markers, while HOTAIR enhanced cells displayed a lowest expression of mature hepatic markers ( $n=3, P<0.05$ ) (Figure $3 \mathrm{C}$ ). As to as for Urea production, Alb production and Alt production, DAPTinduced cells exhibit a significantly higher expression than the other two groups $(n=3, P<0.05)$ (Figure 3D)

\section{Notch inhibition alleviates the carcinogenesis tendency of malignantly transformed LNSCs in vivo}

In previous assays, the in vitro tumor characteristic changes of proliferation, invasive, migration, cell markers,

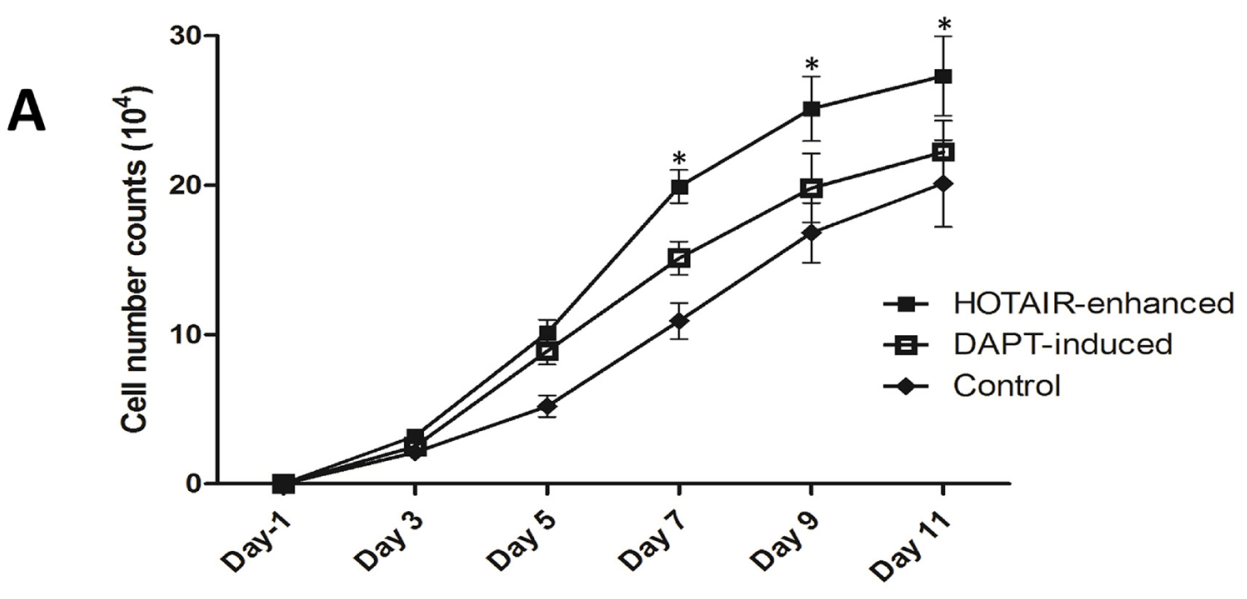

B

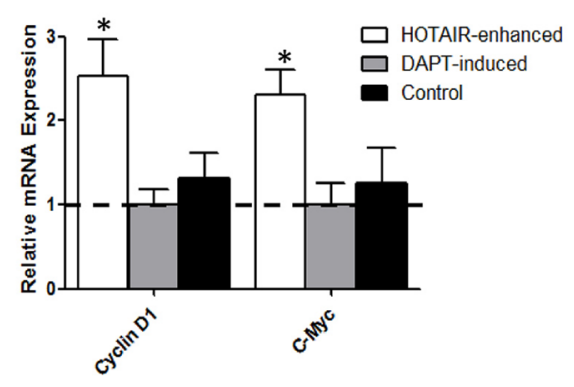

C

$\beta$-actin

HOTAIR- DAPT- Control

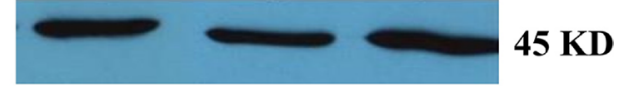

Cyclin D1

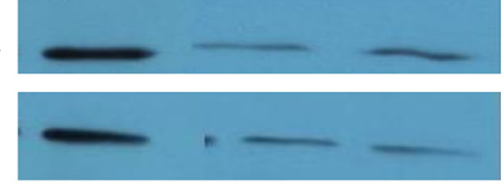

$36 \mathrm{KD}$

57 KD

Figure 1: Notch inhibition could reverse the high proliferative status of malignantly transformed LNSCs. (A) MTT assays showed that proliferative speed was similar in three groups at the early stage. From the 4th day, the number of cells in HOTAIR enhanced group was much higher than that the other two groups $(n=3, P<0.05)$. Nevertheless, no significant difference was observed between DAPT-induced and control group $(n=3, P>0.05)$. (B) At mRNA level, DAPT induced cells presented a lowest Cyclin D1 and c-Myc expression, and was set up as baseline. Cyclin D1 expression in HOTAIR enhanced group were 2.53-fold $(2.53 \pm 0.43, n=3, P<$ $0.05)$ and 1.92-fold $(1.92 \pm 0.30, n=3, P>0.05)$ higher than DAPT induced and control group respectively. As to C-Myc, the xpression rate is 2.31 -fold $(2.31 \pm 0.30, n=3, P<0.05)$ and 1.83 -fold $(1.83 \pm 0.41, n=3, P>0.05)$. (C) WB results showed that the cellular cycle gene Cyclin D1 and proliferative gene c-Myc were downregulated in DAPT-induction or under-expressed in normal cells spontaneously $(n=3, P<0.05)$ at protein level. 
and hepatic function were tested. Tumorigenesis in immunedeficient mice was recommended as the "smoking gun" for cancer formation and for evaluation of anti-tumor therapy. To test the tumorigenic ability of cells from each group, equal numbers of cells were injected sub-percutaneously into mice. The results showed that HOTAIR-enhanced cells developed visible tumors on every nude mouse, whereas no visible tumor was observed on the mice which was injected with LNSCs. Meanwhile, the cells handled with DAPTinducing formed a much smaller tumor than that formed in HOTAIR-enhanced cells (Figure 4A).

\section{The molecular mechanism of Notch inhibition in differentiation inducing therapy}

To explore the molecular mechanism behind the differentiation inducing therapy, the EMT markers E-cadherin and $\beta$-catenin was detected by WB. The results suggested that MET marker E-cadherin expression was upregulated not only at the mRNA level (Figure 4C) but also at the protein level (Figure 4B) in DAPT-induced cells. Oppositely, EMT marker $\beta$-catenin was up-regulated at both mRNA level (Figure 4C) and protein level (Figure 4B) in HOT AIR-enhanced cells. HOTAIR enhancing can lead to E-cadherin degraded and $\beta$-catenin up-regulated, which resulted in EMT, while DAPT differentiation inducing therapy could reverse this tendency and trigger the MET processing.

\section{DISCUSSION}

Where and how LCSCs derive from is the core problem of HCC therapy. It is believed that LCSCs originate from dedifferentiation of mature hepatocytes or differentiation arrest of liver normal stem cells (LNSCs) with many molecules involved in such as Hippo and Notch signaling pathway. $\mathrm{Mu}$ et al. found that AFP-, A6-, and CK19-positive cells within HCCs may be hepatocyte-derived cells that underwent dedifferentiation, and the progenitor signature of HCCs does not reflect progenitor origin, but dedifferentiation of hepatocytederived tumor cells [16]. Under specific conditions, hepatocytes present multi-plasticity and dedifferentiate into biliary cells and even immature progenitors. This dedifferentiation ability results in the presentation of an advantage for the tumor [17]. Another finding indicates that loss of Hippo-pathway signaling and upregulation of YAP drive the mature hepatocytes to dedifferentiate into progenitor characteristics [18]; what is more, transgenic mice with YAP enhanced results in increased hepatocyte dedifferentiation, cell migration, EMT, and malignant transformation of LNSCs [19]. It is of great interest that the dedifferentiation of HCCs may predict a worse prognosis [20]. Meanwhile, a quite different idea believes that LCSCs arise out of differentiation and/or maturation arrest of LNSCs [21]. Chiba et al. transferred Bmil and Wnt/beta-catenin into c-Kit ${ }^{(-)} \mathrm{CD} 29^{(+)} \mathrm{CD} 49 \mathrm{f}^{(+/ \text {low })} \mathrm{CD} 45^{(-)}$

\section{A}

HOTAIR-enhanced DAPT-induced
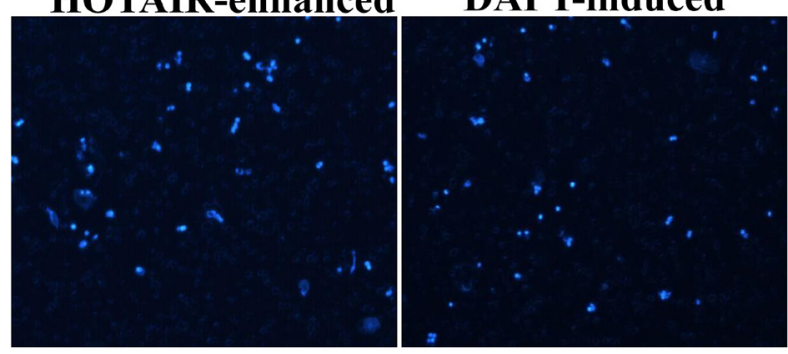

B

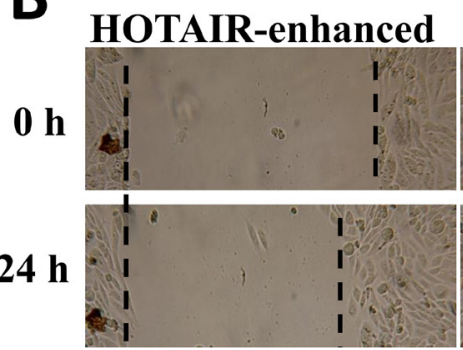

DAPT-induced

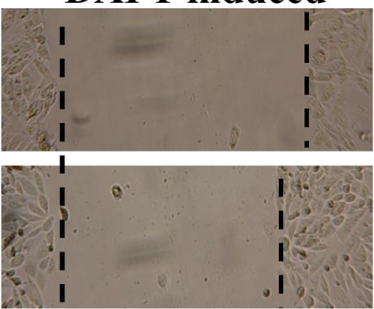

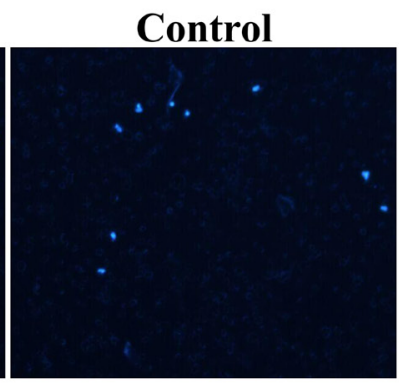

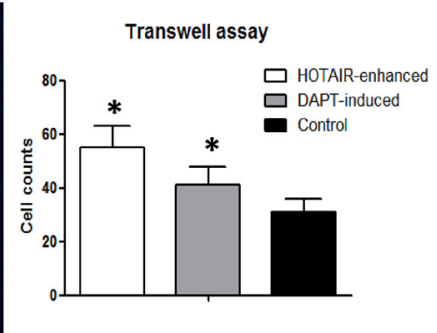

\section{Control}

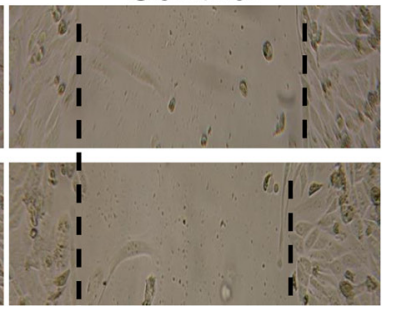

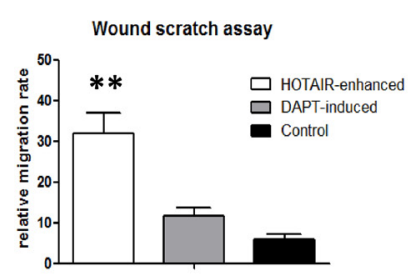

Figure 2: Notch inhibition neutralizes the high invasive status of malignantly transformed LNSCs. (A) Transwell assays showed that the number of trespassed cells in HOTAIR enhanced group was 55/PHF (55 $\pm 8, n=3, P<0.05), 41 / \mathrm{PHF}(41 \pm 7, n=3, P<$ $0.05)$ in DAPT induced group, and 31/PHF $(31 \pm 5)$ in control group. (B) Scratch wound healing assays indicated that the gained migration ability of HOTAIR-enhanced cells could be absolutely counteracted by DAPT inducing $(n=3, P<0.001)$. 
Ter-119(-) hepatic stem/progenitor cells and these cells finally initiate hepatic tumors [22]. With P53 gene deleted in LNSCs, Suzuki et al. suggested that these transgenic cells can result in the formation of liver tumors [23]. In our previous study, suppressing the anti-oncogene Tg737 and miRNA-200a, or enhanced oncogene miRNA-10b and HOTAIR could lead LNSCs to malignant transformation by promoting the cell-cycle progressing and differentiation arrest respectively [24-26].

No matter which theory above is more applicable to the real pathologic procedure of HCC initiation, differentiation-inducing therapy can target both dedifferentiation and differentiation arrest. Although differentiation-inducing therapy has first been administrated in hematologic tumors, recent studies have applied this strategy to liver cancer. All-trans-retinoic acid is powerful weapon for leukemia. However in liver cancer, all-transretinoic acid can alleviate the dedifferentiation effect which is resulted from the upregulated expression of both TGF- $\beta 1$ and CD147, and play a differentiation-inducing effect [27]. Zhang et al. demonstrated that all-trans-retinoic acid can not only contribute to differentiation-inducing therapy function, can also boost the chemo-sensitive of HCCs [4]. Xie et al. illustrated that the core differentiation factors HNF- $1 \alpha$ and $-4 \alpha$ were absent in HCC and compensative upregulation of these absent factors can induce CD133 ${ }^{(+)}$ LCSCs differentiation into mature hepatocytes [8, 28]. Oncostatin M (OSM) is known to be able to induce the

\section{A HOTAIR-enhanced DAPT-induced}
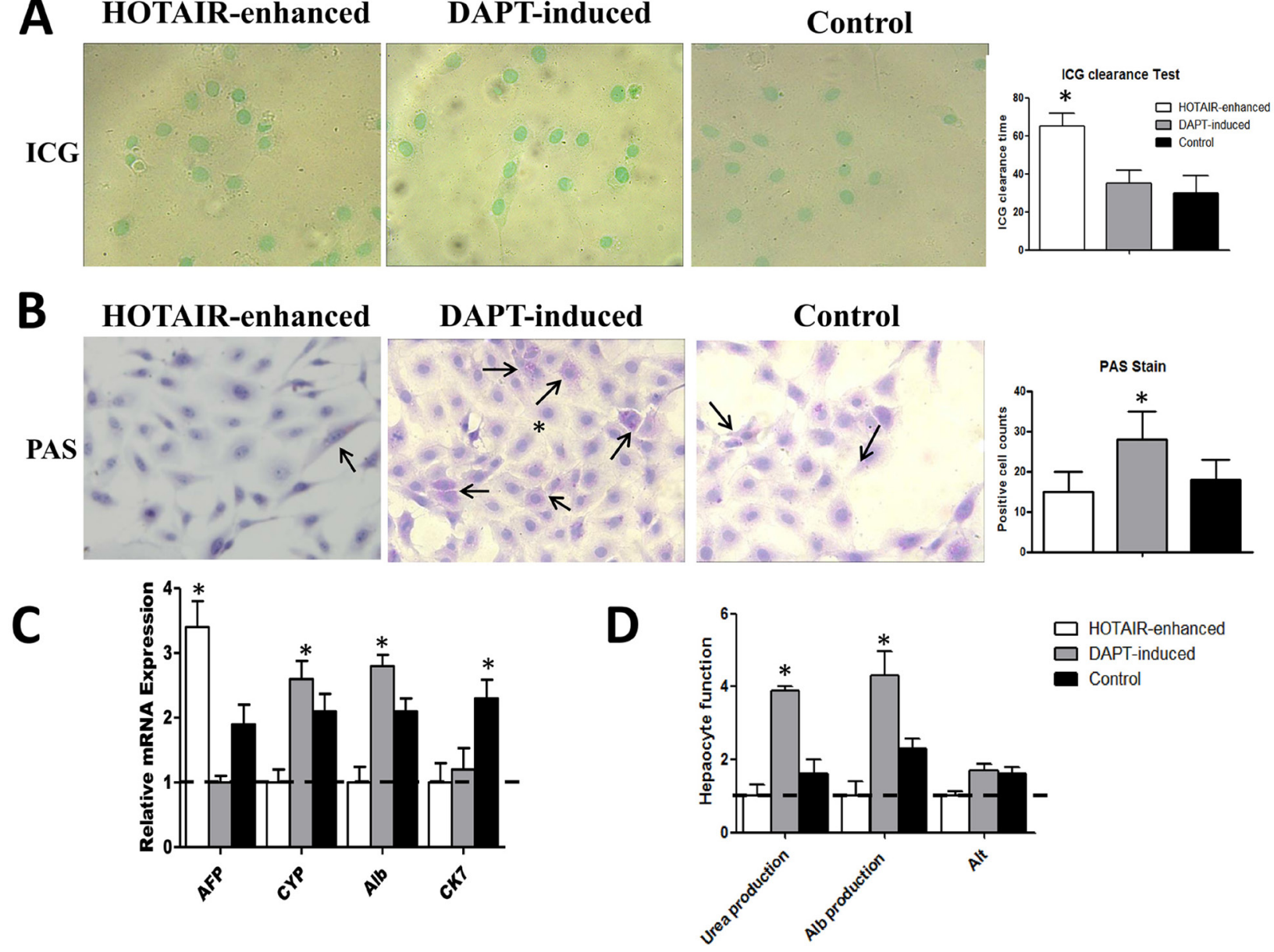

Figure 3: Notch inhibition drives malignantly transformed LNSCs into mature hepatocytes. (A) In all three groups, cells were able to intake the ICG dye after incubation. However, the ICG clearance time is different in each group. In HOTAIR enhanced group, the clearance time is 65 minutes $(65 \pm 7, n=3, P<0.05), 35$ minutes $(35 \pm 7, n=3, P>0.05)$ in DAPT-induced group and 30 minutes $(30 \pm 9)$ in control group. The difference between DAPT-induced group and control group was not significant. (B) The number of PASpositive cells was $28 / \mathrm{HPF}(28 \pm 7, n=10, P<0.05)$ in DAPT-induced group, and it is significantly higher than $15 / \mathrm{HPF}(15 \pm 5, n=10$, $P>0.05)$ in HOTAIR enhanced group and 18/HPF $(18 \pm 5)$ in control group $(n=10, P<0.001)$. (C) The lowest expression sample was set up as baseline. Immature hepatic marker AFP was 3.3-fold $(3.3 \pm 0.4, n=3, P<0.05)$ and 1.8 -fold $(1.8 \pm 0.3, n=3, P>0.05)$ higher than that in DAPT-induction and LNSCs respectively. CK-7 expression was highest expressed in LNSCs $(n=3, P<0.05)$. The patterns of mature hepatic marker, CYP and Alb expression were similar. DAPT-induced cells presents a highest expression of these mature hepatic markers, while HOTAIR enhanced cells displayed a lowest expression of mature hepatic markers $(n=3, P<0.05)$. (D) As to as for Urea production, Alb production and Alt production, DAPT-induced cells exhibit a significantly higher expression than the other two groups $(n$ $=3, P<0.05)$. Black arrow showed the PAS-positive cells. 
LNSCs into hepatocytes. However in the $\operatorname{EpCAM}^{(+)}$stem phenotype HCCs, OSM can decrease the stemness-related gene expression, such as EpCAM, $\alpha$-fetoprotein and CK19 [7]. Similar with OSM, in our previous study, it was demonstrated that Notch inhibition could promote FLSPCs differentiation into hepatocytes, and these differentiated cells presented multiple hepatic functions $[10,11]$. It is true that differentiation-inducing therapy is novel therapeutic method for solid tumor such as HCC. We believe that the feasibility of differentiation-inducing therapy lies on several facts. First, many antitumor reagents, like miR-148a, DMSO and Notch signal inhibitor DAPT, are also known as differentiation inducer. Jung [29] identified miR-148a as an inducer of hepatic differentiation, while MiR-148a-mimetic treatment in vivo suppressed tumor growth, reduced tumor malignancy in liver. Dimethyl sulfoxide (DMSO) can enhance the chemo-sensitive response to chemotherapeutic agents in HCC [30], while DMSO can also accelerate the hepatogenic procedures of adipose tissue-derived mesenchymal stem cells [31]. Similar patterns happen to DAPT $[32,33]$. In this study, we are trying to figure out whether inhibition of Notch can play differentiation-inducing therapy and reverse the malignant transformation procedures.

Although differentiation-inducing therapy will not kill the HCCs or LCSCs directly, it can down-regulate the stemness-related cancer markers, lower the proliferative status, alleviate the invasive characteristic, or attenuate the metastasis tendency. Previous studies focus on the fact that differentiation therapy can down-regulate cancer markers, while the fact that the hepatic function recover after differentiation therapy is the more important. In this study, we lay our emphasis not only on the cancer markers, but also on the recovery of the hepatocyte function. On cancer characteristic, inhibition Notch could decrease the proliferation through downregulation of oncogene c-Myc and Cyclin D1. It can reverse the invasive status, terminate metastasis tendency, and decrease tumorigenesis ability. More meaningfully, it will help the malignantly

A
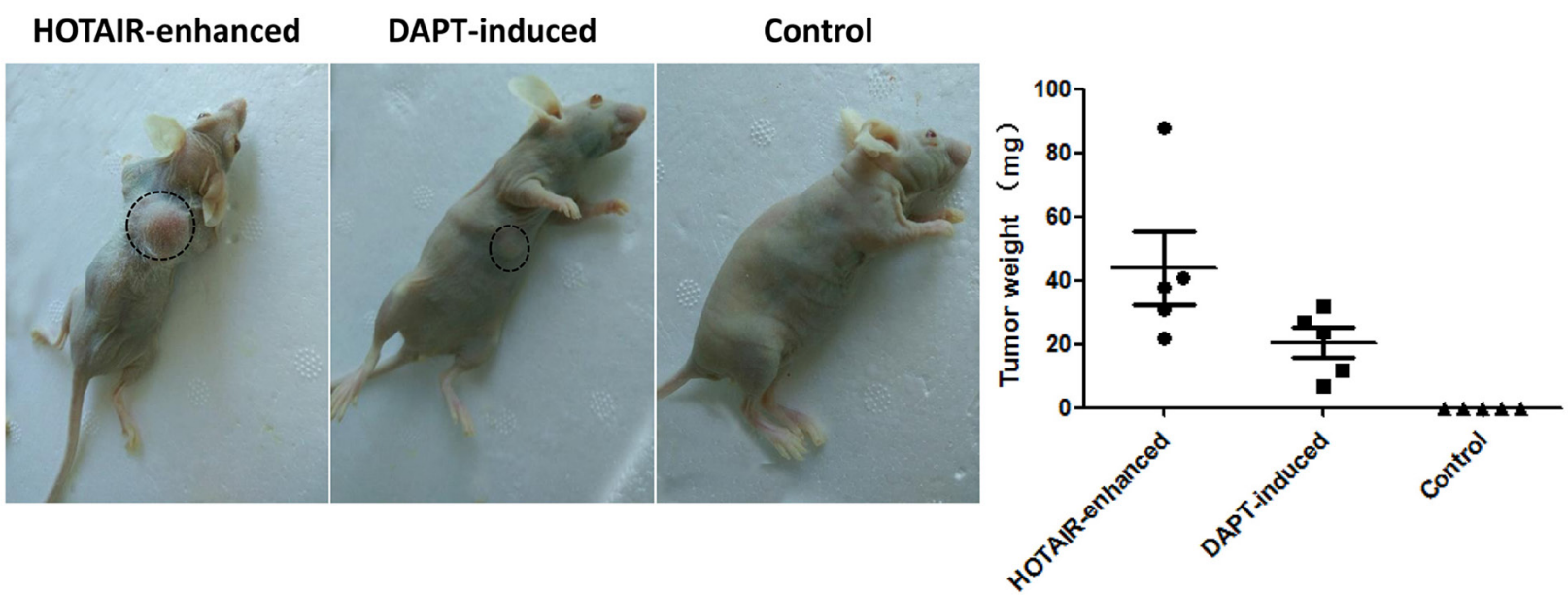

B

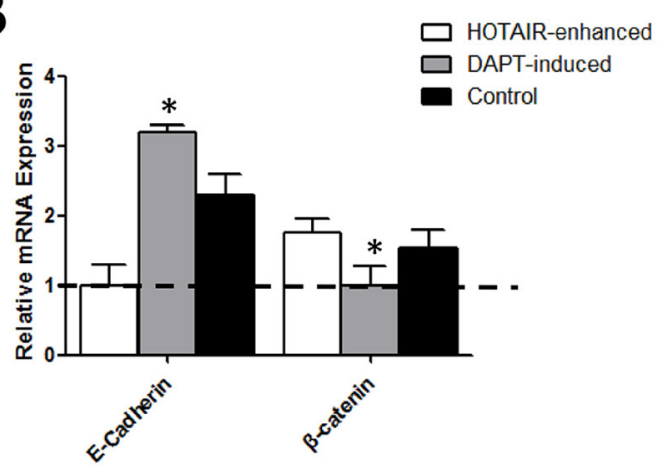

C

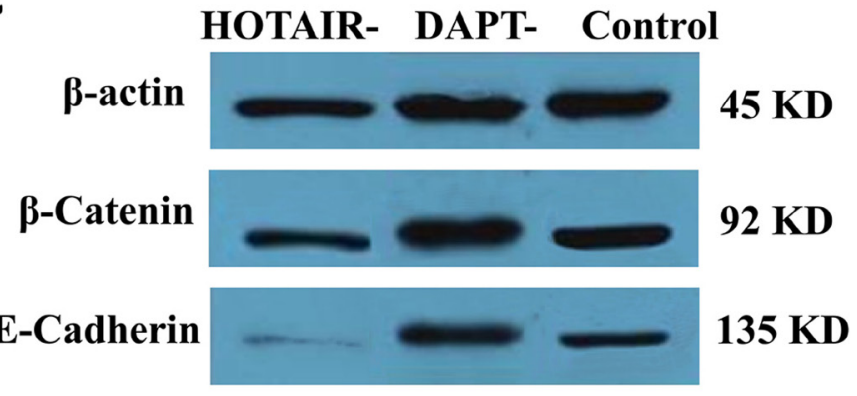

Figure 4: Notch inhibition alleviates the carcinogenesis tendency of malignantly transformed LNSCs via EMT. (A) The results showed that HOTAIR-enhanced cells developed visible tumors on every nude mouse, whereas no visible tumor was observed on the mice which were injected with LNSCs. Meanwhile, the cells handled with DAPT-inducing formed a much smaller tumor than that formed in HOTAIR-enhanced cells. (B, C) The results suggested that the MET markers E-Cadherin expression was up-regulated not only at the mRNA level (3.2-fold higher than baseline, $3.2 \pm 0.1, n=3, P<0.05)$ but also at the protein level in DAPT-induced cells. Oppositely, EMT marker $\beta$-catenin was up-regulated at both mRNA level (1.8-fold higher than baseline, $1.8 \pm 0.3, n=3, P<0.05)$ and protein level in HOTAIR-enhanced cells. The black cycle indicated the neoplastic tumor. The lowest expression sample was set up as baseline. 
transformed cells to regain the mature hepatic function of glucagon synthesis and ICG elimination. Clearance of ICG is an important factor to evaluate the hepatic function. Additionally, ICG was found highly loaded at tumor tissue, and helped to identify the margin of the tumor [34]. Because the HCCs are not able to eliminate the ICG, it will accumulate in the tumor tissue; what is more, the accumulation of ICG is correlated with the differentiation status of HCCs [29, 30]. Well-differentiated HCCs show light and uniform fluorescence of ICG in the cancerous tissue, while poorly differentiated HCCs show strong and rim-type fluorescence around the tumor [35]. Our results demonstrated that Notch inhibition was able to accelerate the clearance speed of ICG in malignantly transformed cells due to the promotion of the differentiation status. It is a sign for both LNSCs differentiation into mature hepatocytes and LCSCs response to differentiation-inducing therapy.

The mechanisms behind the malignant transformation and differentiation-inducing therapy are complicated. Many evidences give clues that EMT process is involved in malignant transformation and drive the LNSCs into LCSCs via either dedifferentiation [36] or differentiation arrested (red arrow in Figure 5) [37, 38], while the MET process is the initiative factors for LNSCs maturation and differentiation (black arrow in Figure 5). Differentiation-inducing therapy is novel therapeutic strategy for solid tumor management. It has been proved that many molecules and signaling pathways are involved in such as HNF families, miRNA families, Wnt signaling pathways $[39,40]$. In this study, we found that the antitumor effects of Notch inhibition may lie not only on killing the HCCs or LCSCs directly, it can also induce the LCSCs differentiation into mature hepatocytes via MET progress and downgrade the malignancy. However, there are still some unsolved paradoxes in differentiationinducing therapy. For one thing, inhibition of Notch promotes LNSCs differentiate into hepatocytes, and arrest differentiation of cholangiocytes [41, 42], while Notch activation drives carcinogenesis, development and progression of cholangiocarcinoma in mice and human [43-45]. The paradoxical role of Notch in cholangiocytes differentiation and cholangio-carcinogenesis is our future research interests. For another, although it is sure that the destination of differentiation inducing therapy is mature hepatocytes, whether LNSC is a transition state between LCSC and mature hepatocyte, or differentiation inducing therapy can directly drive LCSCs to differentiate into mature hepatocytes it is still unknown.

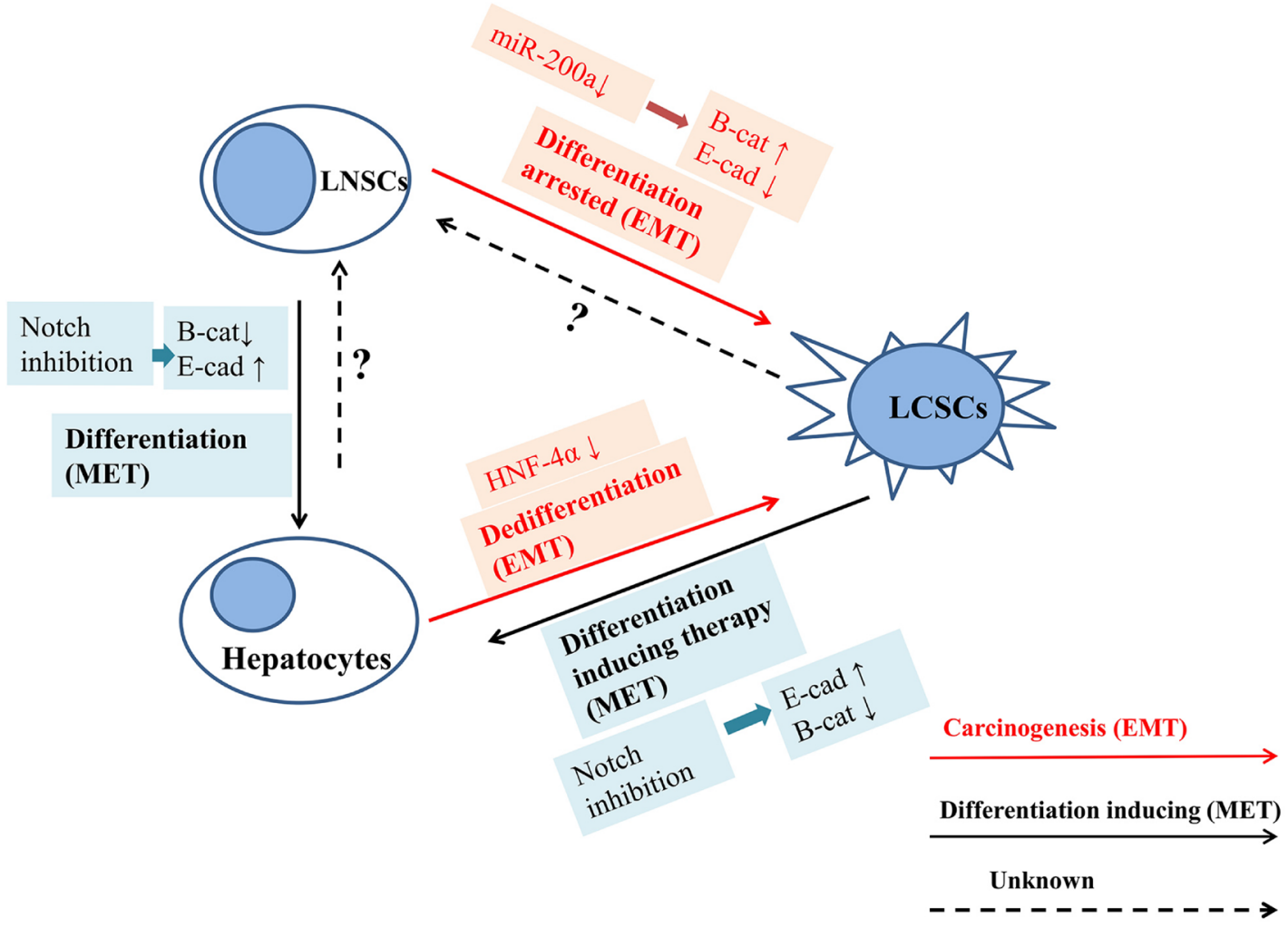

Figure 5: The mechanism of differentiation inducing therapy. EMT process is involved in malignant transformation and drives the LNSCs into LCSCs via either dedifferentiation or differentiation arrested, while the MET process is the initiative factors for LNSCs maturation and differentiation. It has been proved that many molecules and signaling pathways are involved in such as HNF families, miRNA families, and Wnt signaling pathways. The anti-tumor effects of Notch inhibition may lie not only on killing the HCCs or LCSCs directly, it can also induce the LCSCs differentiation into mature hepatocytes via MET progress and downgrade the malignancy. The red arrow shows the process of EMT or malignant transformation, and the black arrow indicates the process of differentiation inducing therapy and MET. 
Table 1: Primers sequences for QRT-PCR

\begin{tabular}{|c|c|c|c|}
\hline Name & Accession number & Primer sequences & Product size (bp) \\
\hline AFP F & \multirow[t]{2}{*}{ NM_012493.2 } & ctgta tgctc ccacc attat tt & \multirow{2}{*}{109} \\
\hline AFP R & & ttgat gctct ctttg tctgg aa & \\
\hline ALB F & \multirow[t]{2}{*}{ NM_134326.2 } & gacaa agcag cctgc ctgac & \multirow{2}{*}{174} \\
\hline ALB R & & ttctg cgaac tcagc attgg & \\
\hline$\beta$-Catenin F & \multirow[t]{2}{*}{ NM_053357.2 } & actcc aggaa tgaag gcgtg & \multirow[t]{2}{*}{109} \\
\hline$\beta$-Catenin $\mathrm{R}$ & & gaact ggtca gctga accga & \\
\hline CYP & \multirow[t]{2}{*}{ NM_013105.2 } & cattc ctcac gecag tatat ga & \multirow{2}{*}{198} \\
\hline CYP & & cggat aggge tgtat gagat tc & \\
\hline CK7 F & \multirow[t]{2}{*}{ NM_001047870.1 } & aggaa cagaa gtcag ccaag ag & \multirow{2}{*}{210} \\
\hline CK7 R & & gcaac acaaa ctcat tctca gc & \\
\hline C-Myc F & \multirow[t]{2}{*}{ NM_012603.2 } & cgagc tgaag cgtag ctttt & \multirow[t]{2}{*}{170} \\
\hline C-Myc R & & ctcge cgttt cctca gtaag & \\
\hline Cyclin-D1 F & NM_171992.4 & gcgta ccctg acacc aatct & 233 \\
\hline Cyclin-D1 R & \multirow{3}{*}{ NM_017008.4 } & ggctc cagag acaag aaacg & \\
\hline Gapdh F & & atggt ggtga agacg ccagt a & \multirow{2}{*}{143} \\
\hline Gapdh R & & ggcac agtca agget gagat $g$ & \\
\hline
\end{tabular}

\section{MATERIALS AND METHODS}

\section{Cell isolation, culture and transfection}

Adult experimental F344 rats were purchased from West China Medical School of China. The Research Animal Ethics Committee at the Chengdu Military General Hospital approved the animal study protocol (animal protocol number CMGH-2013-023).

The isolation and purification of LNSCs were performed as described previously [46]. The cells were grouped according to the transfection conditions as follows: HOTAIR enhanced group and HOTAIR enhanced plus Notch inhibition group (DAPT group, with a DAPT concentration of $1 \mu \mathrm{M})$. The cells in negative control group were treated with dimethyl sulfoxide (DMSO) (control group). Cells in each group were cultured in Williams' E medium (Sigma, USA) with $15 \%$ (v/v) fetal bovine serum (Invitrogen, USA). The cells were incubated under $5 \%(\mathrm{v} / \mathrm{v}) \mathrm{CO}_{2}$ at $37^{\circ} \mathrm{C}$.

In HOTAIR enhanced group, pCDNA/HOTAIR was transfected into LNSCs using Lipofectamine 2000 according to the manufacturer's instructions.

\section{MTT assay}

The cellular proliferative ability from each group was determined through the 3-(4,5-dimethylthiazol-2yl)-2,5-diphenyltetrazolium bromide (MTT) assay. The cells from each group were cultured in 96-well plates at a concentration of $5 \times 10^{3}$ cells/well. Sterile PBS including $20 \mu \mathrm{l} \mathrm{MTT} \mathrm{(final} \mathrm{concentration} \mathrm{of} 5 \mathrm{mg} / \mathrm{ml}$ ) (Sigma, USA) was added to each well and incubated at room temperature for $4 \mathrm{~h}$, and a continuous detection were made at 1, 3, 5, 7, 9, and 11 days of culture. To fully dissolve the crystal, $150 \mu \mathrm{l}$ DMSO (Invitrogen, USA) was added into each well. The optical density was measured at a wavelength of $490 \mathrm{~nm}$ with a Bio-Rad 680 microplate reader (Bio-Rad, USA).

\section{Transwell migration assay}

A total cellular number of $5 \times 10^{3}$ in $500 \mu$ of serumfree Williams'E medium from each group were seeded into an 8- $\mu \mathrm{m}$ pore-size polycarbonate membrane Boyden chamber, which was inserted into a transwell apparatus (Costar, MA, USA). Additional $500 \mu \mathrm{l}$ Williams' E medium containing higher FBS (15\%) was added into the lower layer of 24 -well plate. After incubated at $37^{\circ} \mathrm{C}$ for a day, the trespassed cells were fixed in $95 \%$ ethanol for $5 \mathrm{~min}$, stained with DAPI $(1 \mu \mathrm{g} / \mathrm{mL})$, rinsed in PBS for $3 \times 5$ mins, and observed via fluorescence microscopy (Olympus, Japan).

\section{Scratch wound healing assays}

A total number of $5 \times 10^{3}$ cells from each group were added into 6-well plate and incubated overnight in FBS free medium. Pipette tips were used to make wounds on the attached growing cells. The wound healing procedure was observed for $24 \mathrm{~h}$, and dead cells were gently washed off before photographs were taken.

\section{Real time polymerase chain reaction (RT-PCR)}

RNA was harvested using Trizol (Invitrogen, USA), according to the manufacturer's instructions. The 
cDNA synthetization was performed as Primescript RT reagent Kit (TAKARA, China). The specific primers were exhibited in Table 1. PCR reactions were performed as following: template denaturation at $95^{\circ} \mathrm{C}$ for $1 \mathrm{~min}$, primer annealing at $60^{\circ} \mathrm{C}$ for $1 \mathrm{~min}$, primer extension at $72^{\circ} \mathrm{C}$ for $2 \mathrm{~min}$ ( 35 cycles), and $72^{\circ} \mathrm{C}$ for $10 \mathrm{~min}$ as an extra cycle of elongation.

\section{Protein extraction and western-blot (WB)}

The cells were pyrolysis in RIPA buffer containing protease inhibitors. The lysates were centrifuged at $10,000 \mathrm{rpm}$ for $10 \mathrm{~min}$ at $4^{\circ} \mathrm{C}$. After centrifuge, the supernatant were collected. Total of $50 \mu \mathrm{g}$ protein was uploaded to sodium dodecyl sulfate polyacrylamide gel electrophoresis and blotted onto a PVDF membrane. The membrane was blocked using 5\% fat-free milk at room temperature for $2 \mathrm{~h}$. The concentration of antibody for incubated is as following: anti-E-cadherin (1:200), antiCyclin D1 $(1: 1,000)$, anti-C-Myc $(1: 1,000)$, anti- $\beta$-catenin (1:500), anti- $\beta$-actin $(1: 1,000)$ (Sigma, USA) at $4^{\circ} \mathrm{C}$ for overnight. After three washes for 5-min in TBST, the membrane was incubated in peroxidase-conjugated goat anti-mouse/rabbit IgG or peroxidase-conjugated rabbit anti-goat IgG (Abcam, US) for $2 \mathrm{~h}$ at room temperature. An electrogenerated chemiluminescence system was used to visualize the proteins.

\section{Xenograft mouse model}

About $5 \times 10^{7}$ cells from each group were subcutaneously injected into the nude mice on the back separately. All mice were sacrificed 60 days after injection, and the weight of the tumor was detected.

\section{Statistical analysis}

All of the experiments were independently replicated triply. Statistical analysis was performed using SPSS Version 19.0 (SPSS, USA). Continuous variables were evaluated using Fisher's exact $t$-test or MannWhitney $u$-test. Discrete variables were assessed using the Chi-square test. Two-tailed $P$ values $<0.05$ were considered statistical significance.

\section{Abbreviations}

alpha-fetoprotein: AFP; high power field: HPF; CT: cycle threshold; CYP: cytochrome P450; CK: cytokeratin; polymerase chain reaction: PCR; DAPT: N-[N-(3: 5-Difluorophenacetyl)-L-alanyl]-S-phenylglycine t-butyl ester; LCSCs: liver cancer stem cells; LNSCs: liver normal stem cells; ICG: indocyanine green; PAS: Periodic Acid Schiff's staining; ncRNA: non-coding RNA; EMT: epithelial-mesenchymal transition; MET: mesenchymalepithelial transition; HOTAIR: HOX transcript antisense
RNA; HNF: hepatocyte nuclear factor; DMSO: dimethyl sulfoxide.

\section{ACKNOWLEDGMENTS}

This work was granted by the Chinese National Natural Science Foundation (grant no. 81502696 and grant no. 81302168). We thank JiongRan Medical Art Studio for the image processing and schematic picture.

\section{CONFLICTS OF INTEREST}

All authors have no conflicts of interest.

\section{REFERENCES}

1. Guinea-Viniegra J, Zenz R, Scheuch H, Jimenez M, Bakiri L, Petzelbauer P, Wagner EF. Differentiation-induced skin cancer suppression by FOS, p53, and TACE/ADAM17. J Clin Invest. 2012; 122:2898-910. https://doi.org/10.1172/JCI63103.

2. Mishra R, Kaur G. Aqueous ethanolic extract of Tinospora cordifolia as a potential candidate for differentiation based therapy of glioblastomas. PLoS One. 2013; 8:e78764. https://doi.org/10.1371/journal.pone.0078764.

3. Zhang Z, Zhou Y, Qian H, Shao G, Lu X, Chen Q, Sun X, Chen D, Yin R, Zhu H, Shao Q, Xu W. Stemness and inducing differentiation of small cell lung cancer NCI-H446 cells. Cell Death Dis. 2013; 4:e633. https://doi.org/10.1038/ cddis.2013.152.

4. Zhang Y, Guan DX, Shi J, Gao H, Li JJ, Zhao JS, Qiu L, Liu J, Li N, Guo WX, Xue J, Zhou FG, Wu MC, et al. Alltrans retinoic acid potentiates the chemotherapeutic effect of cisplatin by inducing differentiation of tumor initiating cells in liver cancer. J Hepatol. 2013; 59:1255-63. https:// doi.org/10.1016/j.jhep.2013.07.009.

5. You N, Zheng L, Liu W, Zhong X, Wang W, Li J. Proliferation inhibition and differentiation induction of hepatic cancer stem cells by knockdown of BC047440: a potential therapeutic target of stem cell treatment for hepatocellular carcinoma. Oncol Rep. 2014; 31:1911-20. https://doi.org/10.3892/or.2014.3043.

6. Mishra PJ, Merlino G. MicroRNA reexpression as differentiation therapy in cancer. J Clin Invest. 2009; 119:2119-23. https://doi.org/10.1172/JCI40107.

7. Yamashita T, Honda M, Nio K, Nakamoto Y, Yamashita T, Takamura H, Tani T, Zen Y, Kaneko S. Oncostatin m renders epithelial cell adhesion molecule-positive liver cancer stem cells sensitive to 5-Fluorouracil by inducing hepatocytic differentiation. Cancer Res. 2010; 70:4687-97. https://doi.org/10.1158/0008-5472.CAN-09-4210.

8. Zeng X, Lin Y, Yin C, Zhang X, Ning BF, Zhang Q, Zhang JP, Qiu L, Qin XR, Chen YX, Xie WF. Recombinant adenovirus carrying the hepatocyte nuclear factor-1alpha gene inhibits hepatocellular carcinoma xenograft growth 
in mice. Hepatology. 2011; 54:2036-47. https://doi. org/10.1002/hep.24647.

9. Tanimizu N, Miyajima A. Notch signaling controls hepatoblast differentiation by altering the expression of liverenriched transcription factors. J Cell Sci. 2004; 117:3165-74. https://doi.org/10.1242/jcs.01169.

10. Wang T, Chen T, Liang HY, Yan HT, Lin N, Liu LY, Luo H, Huang Z, Li NL, Liu WH, Tang LJ. Notch inhibition promotes fetal liver stem/progenitor cells differentiation into hepatocytes via the inhibition of HNF-1beta. Cell Tissue Res. 2014; 357:173-84. https://doi.org/10.1007/ s00441-014-1825-9.

11. Wang T, You N, Tao K, Wang X, Zhao G, Xia N, Li N, Tang L, Liu W, Dou K. Notch is the key factor in the process of fetal liver stem/progenitor cells differentiation into hepatocytes. Dev Growth Differ. 2012; 54:605-17. https:// doi.org/10.1111/j.1440-169X.2012.01363.x.

12. Bi P, Yue F, Karki A, Castro B, Wirbisky SE, Wang C, Durkes A, Elzey BD, Andrisani OM, Bidwell CA, Freeman JL, Konieczny SF, Kuang S. Notch activation drives adipocyte dedifferentiation and tumorigenic transformation in mice. J Exp Med. 2016; 213:2019-37. https://doi.org/10.1084/ jem.20160157.

13. Kim W, Khan SK, Gvozdenovic-Jeremic J, Kim Y, Dahlman J, Kim H, Park O, Ishitani T, Jho EH, Gao B, Yang Y. Hippo signaling interactions with Wnt/beta-catenin and Notch signaling repress liver tumorigenesis. J Clin Invest. 2017; 127:137-52. https://doi.org/10.1172/JCI88486.

14. Chen J, Chang H, Peng X, Gu Y, Yi L, Zhang Q, Zhu J, Mi M. 3,6-dihydroxyflavone suppresses the epithelialmesenchymal transition in breast cancer cells by inhibiting the Notch signaling pathway. Sci Rep. 2016; 6:28858. https://doi.org/10.1038/srep28858.

15. Zhao ZL, Zhang L, Huang CF, Ma SR, Bu LL, Liu JF, Yu GT, Liu B, Gutkind JS, Kulkarni AB, Zhang WF, Sun ZJ. NOTCH1 inhibition enhances the efficacy of conventional chemotherapeutic agents by targeting head neck cancer stem cell. Sci Rep. 2016; 6:24704. https://doi.org/10.1038/ srep24704.

16. Mu X, Espanol-Suner R, Mederacke I, Affo S, Manco R, Sempoux C, Lemaigre FP, Adili A, Yuan D, Weber A, Unger K, Heikenwalder M, Leclercq IA, Schwabe RF. Hepatocellular carcinoma originates from hepatocytes and not from the progenitor/biliary compartment. J Clin Invest. 2015; 125:3891-903. https://doi.org/10.1172/JCI77995.

17. Yanger K, Zong Y, Maggs LR, Shapira SN, Maddipati R, Aiello NM, Thung SN, Wells RG, Greenbaum LE, Stanger BZ. Robust cellular reprogramming occurs spontaneously during liver regeneration. Genes Dev. 2013; 27:719-24. https://doi.org/10.1101/gad.207803.112.

18. Yimlamai D, Christodoulou C, Galli GG, Yanger K, PepeMooney B, Gurung B, Shrestha K, Cahan P, Stanger BZ, Camargo FD. Hippo pathway activity influences liver cell fate. Cell. 2014; 157:1324-38. https://doi.org/10.1016/j. cell.2014.03.060.
19. Nishio M, Sugimachi K, Goto H, Wang J, Morikawa T, Miyachi Y, Takano Y, Hikasa H, Itoh T, Suzuki SO, Kurihara H, Aishima S, Leask A, et al. Dysregulated YAP1/TAZ and TGF-beta signaling mediate hepatocarcinogenesis in Mob1a/1b-deficient mice. Proc Natl Acad Sci USA. 2016; 113: E71-80. https://doi.org/10.1073/pnas.1517188113.

20. Lee JS, Heo J, Libbrecht L, Chu IS, Kaposi-Novak P, Calvisi DF, Mikaelyan A, Roberts LR, Demetris AJ, Sun Z, Nevens F, Roskams T, Thorgeirsson SS. A novel prognostic subtype of human hepatocellular carcinoma derived from hepatic progenitor cells. Nat Med. 2006; 12:410-6. https:// doi.org/10.1038/nm1377.

21. Sell S. Cellular origin of cancer: dedifferentiation or stem cell maturation arrest? Environ Health Perspect. 1993; 101:15-26.

22. Chiba T, Zheng YW, Kita K, Yokosuka O, Saisho H, Onodera M, Miyoshi H, Nakano M, Zen Y, Nakanuma Y, Nakauchi H, Iwama A, Taniguchi H. Enhanced self-renewal capability in hepatic stem/progenitor cells drives cancer initiation. Gastroenterology. 2007; 133:937-50. https://doi. org/10.1053/j.gastro.2007.06.016.

23. Suzuki A, Sekiya S, Onishi M, Oshima N, Kiyonari H, Nakauchi H, Taniguchi H. Flow cytometric isolation and clonal identification of self-renewing bipotent hepatic progenitor cells in adult mouse liver. Hepatology. 2008; 48:1964-78. https://doi.org/10.1002/hep.22558.

24. Ye P, Wang T, Liu WH, Li XC, Tang LJ, Tian FZ. Enhancing HOTAIR/MiR-10b Drives Normal Liver Stem Cells Toward a Tendency to Malignant Transformation Through Inducing Epithelial-to-Mesenchymal Transition. Rejuvenation Res. 2015; 18:332-40. https://doi.org/10.1089/rej.2014.1642.

25. Liu J, Ruan B, You N, Huang Q, Liu W, Dang Z, Xu W, Zhou T, Ji R, Cao Y, Li X, Wang D, Tao K, Dou K. Downregulation of miR-200a induces EMT phenotypes and CSC-like signatures through targeting the beta-catenin pathway in hepatic oval cells. PLoS One. 2013; 8:e79409. https://doi.org/10.1371/journal.pone.0079409.

26. You N, Liu W, Zhong X, Ji R, Zhang M, You H, Dou K, Tao K. Tg737 inhibition results in malignant transformation in fetal liver stem/progenitor cells by promoting cell-cycle progression and differentiation arrest. Mol Carcinog. 2012; 51:659-73. https://doi.org/10.1002/mc.20839.

27. Wu J, Lu M, Li Y, Shang YK, Wang SJ, Meng Y, Wang Z, Li ZS, Chen H, Chen ZN, Bian H. Regulation of a TGFbeta1-CD147 self-sustaining network in the differentiation plasticity of hepatocellular carcinoma cells. Oncogene. 2016; 35:5468-79. https://doi.org/10.1038/onc.2016.89.

28. Ning BF, Ding J, Yin C, Zhong W, Wu K, Zeng X, Yang W, Chen YX, Zhang JP, Zhang X, Wang HY, Xie WF. Hepatocyte nuclear factor 4 alpha suppresses the development of hepatocellular carcinoma. Cancer Res. 2010; 70:7640-51. https://doi.org/10.1158/0008-5472. CAN-10-0824.

29. Jung KH, Zhang J, Zhou C, Shen H, Gagea M, RodriguezAguayo C, Lopez-Berestein G, Sood AK, Beretta L. 
Differentiation therapy for hepatocellular carcinoma: Multifaceted effects of miR-148a on tumor growth and phenotype and liver fibrosis. Hepatology. 2016; 63:864-79. https://doi.org/10.1002/hep.28367.

30. Tofilon PJ, Vines CM, Milas L. Enhancement of in vitro chemotherapeutic activity by dimethylsulfoxide. Clin Exp Metastasis. 1985; 3:141-50.

31. Alizadeh E, Zarghami N, Eslaminejad MB, Akbarzadeh A, Barzegar A, Mohammadi SA. The effect of dimethyl sulfoxide on hepatic differentiation of mesenchymal stem cells. Artif Cells Nanomed Biotechnol. 2016; 44:157-64. https://doi.org/10.3109/21691401.2014.928778.

32. Chen Y, Zheng S, Qi D, Zheng S, Guo J, Zhang S, Weng Z. Inhibition of Notch signaling by a gamma-secretase inhibitor attenuates hepatic fibrosis in rats. PLoS One. 2012; 7: e46512. https://doi.org/10.1371/journal.pone.0046512.

33. You K, Sun P, Yue Z, Li J, Xiong W, Wang J. NOR1 promotes hepatocellular carcinoma cell proliferation and migration through modulating the Notch signaling pathway. Exp Cell Res. 2017; 352:375-81. https://doi.org/10.1016/j. yexcr.2017.02.032.

34. Zeng C, Shang W, Wang K, Chi C, Jia X, Fang C, Yang D, Ye J, Fang C, Tian J. Intraoperative Identification of Liver Cancer Microfoci Using a Targeted Near-Infrared Fluorescent Probe for Imaging-Guided Surgery. Sci Rep. 2016; 6:21959. https://doi.org/10.1038/srep21959.

35. Majlesara A, Golriz M, Hafezi M, Saffari A, Wild E, Maier-Hein L, Muller-Stich BP, Mehrabi A. Indocyanine Green Fluorescence Imaging in Hepatobiliary Surgery. Photodiagnosis Photodyn Ther. 2016. https://doi. org/10.1016/j.pdpdt.2016.12.005.

36. Sun H, Tang H, Xie D, Jia Z, Ma Z, Wei D, Mishra L, Gao Y, Zheng S, Xie K, Peng Z. Kruppel-like Factor 4 Blocks Hepatocellular Carcinoma Dedifferentiation and Progression through Activation of Hepatocyte Nuclear Factor-6. Clin Cancer Res. 2016; 22:502-12. https://doi. org/10.1158/1078-0432.CCR-15-0528.

37. Benzoubir N, Mussini C, Lejamtel C, Dos Santos A, Guillaume C, Desterke C, Samuel D, Brechot C, Bourgeade MF, Guettier C. Gamma-smooth muscle actin expression is associated with epithelial-mesenchymal transition and stem-like properties in hepatocellular carcinoma. PLoS One. 2015; 10:e0130559. https://doi.org/10.1371/journal. pone. 0130559 .

38. Mani SA, Guo W, Liao MJ, Eaton EN, Ayyanan A, Zhou AY, Brooks M, Reinhard F, Zhang CC, Shipitsin M,
Campbell LL, Polyak K, Brisken C, et al. The epithelialmesenchymal transition generates cells with properties of stem cells. Cell. 2008; 133:704-15. https://doi. org/10.1016/j.cell.2008.03.027.

39. Zieker D, Buhler S, Ustundag Z, Konigsrainer I, Manncke S, Bajaeifer K, Vollmer J, Fend F, Northoff H, Konigsrainer A, Glatzle J. Induction of tumor stem cell differentiation-novel strategy to overcome therapy resistance in gastric cancer. Langenbecks Arch Surg. 2013; 398:603-8. https://doi. org/10.1007/s00423-013-1058-5.

40. Zhang Y, Song WJ, Zhang FQ, Liu WH, Dou KF. Differentiation-inducing activity of hydroxycamptothecin on cancer stem-like cells derived from hepatocellular carcinoma. Dig Dis Sci. 2011; 56:2473-81. https://doi. org/10.1007/s10620-011-1601-6.

41. Lu J, Zhou Y, Hu T, Zhang H, Shen M, Cheng P, Dai W, Wang F, Chen K, Zhang Y, Wang C, Li J, Zheng Y, et al. Notch Signaling Coordinates Progenitor Cell-Mediated Biliary Regeneration Following Partial Hepatectomy. Sci Rep. 2016; 6:22754. https://doi.org/10.1038/srep22754.

42. Zhang X, Du G, Xu Y, Li X, Fan W, Chen J, Liu C, Chen G, Liu C, Zern MA, Mu Y, Liu P. Inhibition of notch signaling pathway prevents cholestatic liver fibrosis by decreasing the differentiation of hepatic progenitor cells into cholangiocytes. Lab Invest. 2016; 96:350-60. https:// doi.org/10.1038/labinvest.2015.149.

43. Guest RV, Boulter L, Dwyer BJ, Kendall TJ, Man TY, Minnis-Lyons SE, Lu WY, Robson AJ, Gonzalez SF, Raven A, Wojtacha D, Morton JP, Komuta M, et al. Notch3 drives development and progression of cholangiocarcinoma. Proc Natl Acad Sci USA. 2016; 113:12250-55. https://doi. org/10.1073/pnas.1600067113.

44. Che L, Fan B, Pilo MG, Xu Z, Liu Y, Cigliano A, Cossu A, Palmieri G, Pascale RM, Porcu A, Vidili G, Serra M, Dombrowski F, et al. Jagged 1 is a major Notch ligand along cholangiocarcinoma development in mice and humans. Oncogenesis. 2016; 5:e274. https://doi. org/10.1038/oncsis.2016.73.

45. Cigliano A, Wang J, Chen X, Calvisi DF. Role of the Notch signaling in cholangiocarcinoma. Expert Opin Ther Targets. 2017; 21:471-83. https://doi.org/10.1080/14728222.2017.1 310842.

46. Liu WH, Li R, Dou KF. Convenient and efficient enrichment of the CD133+ liver cells from rat fetal liver cells as a source of liver stem/progenitor cells. Stem Cell Rev. 2011; 7:94-102. https://doi.org/10.1007/s12015-010-9119-4. 